\title{
Inhibition of miR-18a-3p reduces proliferation of mesothelioma cells and sensitizes them to cisplatin
}

\author{
RUI SUZUKI ${ }^{1}$, VISHWA JEET AMATYA ${ }^{1}$, KEI KUSHITANI ${ }^{1}$, YUICHIRO KAI ${ }^{1,2}$, \\ TAKAHIRO KAMBARA $^{1}$, YUTARO FUJII ${ }^{1}$ and YUKIO TAKESHIMA ${ }^{1}$ \\ ${ }^{1}$ Department of Pathology, Hiroshima University Graduate School of Biomedical and Health Sciences; \\ ${ }^{2}$ Department of Surgical Oncology, Research Institute for Radiation Biology and Medicine, \\ Hiroshima University, Hiroshima 734-8551, Japan
}

Received October 11, 2019; Accepted February 10, 2020

DOI: $10.3892 / 01.2020 .11504$

\begin{abstract}
Malignant pleural mesothelioma is a notorious human malignancy. Despite combination chemotherapy with cisplatin and pemetrexed, the majority of patients with advanced malignant pleural mesothelioma have a poor prognosis. MicroRNAs (miRNAs/miRs) are short non-coding RNAs that regulate various biological processes by binding to the 3'-untranslated region of target gene mRNAs and suppressing their expression. Since abnormal expression patterns of miRNAs are a common feature in human malignancies, a number of them have been researched as potential therapeutic targets. Our previous study demonstrated that microRNA-18a (miR-18a) is upregulated in mesothelioma cell lines compared with in non-neoplastic mesothelial tissues, but its function remains unclear. In the present study, miRNA inhibitor was transfected into mesothelioma cell lines and then analyzed various cellular functions. Mesothelioma cells transfected with the miR-18a inhibitor exhibited lower proliferation and migration rates compared with cells transfected with a negative control inhibitor in proliferation and wound scratch assays, respectively. Additionally, the present study revealed that downregulation of miR-18a increased mesothelioma cell apoptosis. In a chemosensitivity assay, transfection of the miR-18a inhibitor significantly increased the sensitivity of mesothelioma cells to cisplatin but not to pemetrexed. Therefore, miR-18a may be a potential therapeutic target for mesothelioma resistant to cisplatin.
\end{abstract}

Correspondence to: Professor Yukio Takeshima, Department of Pathology, Hiroshima University Graduate School of Biomedical and Health Sciences, 1-2-3 Kasumi, Minami-ku, Hiroshima 734-8551, Japan

E-mail: ykotake@hiroshima-u.ac.jp

Key words: malignant mesothelioma, microRNA, microRNA-18a, cisplatin, progression

\section{Introduction}

Malignant pleural mesothelioma, an aggressive tumor, is commonly caused by exposure to asbestos with a median latency of 22.8 years (1). The incidence of malignant mesothelioma is predicted to reach a peak around 2020 in Europe (2) and around 2030 in Japan (3), and has not yet declined this century in the United States (4). The incidence of malignant mesothelioma in developing countries is predicted to increase due to their heavy use of asbestos without restriction (5).

Because many cases of malignant pleural mesothelioma are detected at an advanced stage and are thus unresectable by surgery, systemic anticancer chemotherapy is the first choice of treatment. The present standard regimen of chemotherapy for advanced malignant pleural mesothelioma is a combination of pemetrexed and cisplatin; however, the median survival of patients treated with this regimen scarcely exceeds 12 months (6).

MicroRNAs (miRNAs) are short non-coding, single-strand RNAs that suppress gene expression by binding to the 3'-untranslated region of their target mRNAs (7). Recently, several studies reported different miRNAs that play important roles in the pathogenesis of various human cancers as onco-miRNAs or tumor suppressor miRNAs (8-10).

In a previous study, we investigated the expression profiles of miRNAs in mesothelioma cell lines and found some to be significantly down- or upregulated (11). In that study, microRNA-18a (miR-18a) was one of the upregulated miRNAs. Further, miR-18a has been identified as an onco-miRNA associated with many human malignancies including glioblastoma (12), esophageal cancer (13), and non-small cell lung cancer (14). The current study was performed to elucidate the biological function of miR-18a in mesothelioma.

\section{Materials and methods}

Mesothelioma cell lines. Four human mesothelioma cell lines were used in this study. Two cell lines (ACC-MESO1 and ACC-MESO4) were purchased from the RIKEN BioResearch Center (Tsukuba, Japan) (15) and the other two (CRL-5915 and CRL-5946) were purchased from the American Type Culture Collection. Cells were cultured with Roswell Park Memorial 
Institute 1640 medium with GlutaMax added, containing $10 \%$ fetal bovine serum, sodium pyruvate, kanamycin, and fungizone (all purchased from Thermo Fisher Scientific). Cells were maintained in a $5 \% \mathrm{CO}_{2}$ incubator at $37^{\circ} \mathrm{C}$.

Transient transfection of mesothelioma cells. The miR-18a inhibitor (mirVana; has-miR-18a-3p MH12264) and a negative control miRNA inhibitor (mirVana; negative control \#1) were purchased from Thermo Fisher Scientific. Mesothelioma cells at $60-80 \%$ confluence were transfected with $50 \mathrm{nM}$ of the miR-18a or negative control inhibitor using Lipofectamine RNAiMAX (Thermo Fisher Scientific) in Opti-Mem Reduced Serum Medium (Thermo Fisher Scientific) according to the manufacturer's recommended protocols.

Cell proliferation assay. After transfection, mesothelioma cell lines were incubated in growth medium in a 96-well plate. The proliferation rate was determined at 24,48 , and $72 \mathrm{~h}$ with the Cell Titer Glo 2.0 reagent (Promega KK), which measures the number of viable cells relative to their ATP level, using a GloMax Explore microplate reader (Promega) according to the manufacturer's recommended protocols.

Colony formation assay. Mesothelioma cell lines transfected with the miRNA inhibitor or control were seeded into collagen-coated 6-well plates at a density of 500 cells/well and incubated in growth medium for three weeks. Cellmatrix Type I-A (Nitta Gelatin) was used for collagen coating. Colonies were stained by Cell Stain (EMD Millipore) and counted.

Wound scratch assay. The migration ability of mesothelioma cells was analyzed by a wound scratch assay. miRNA inhibitor- and control-transfected cells were incubated in collagen-coated 24-well plates. After making wounds with $1 \mathrm{ml}$ micropipette tips, floating cells were removed by washing twice with fresh growth medium. Microscopic images were obtained at 0,12 , and $24 \mathrm{~h}$ (ACC-MESO1 cells), or at 0,24 , and $48 \mathrm{~h}$ (ACC-MESO4, CRL-5915, and CRL-5946 cells). The wound area was measured using TScratch software (16).

Cell invasion assay. Mesothelioma cell lines were incubated with the miRNA or control inhibitor in FluoroBlok culture inserts with 8- $\mu \mathrm{m}$ pores (BD Biosciences) and coated with Geltrex Matrigel (Thermo Fisher Scientific) according to the manufacturers' protocols. Invaded cells were measured at 24 or $48 \mathrm{~h}$ after incubation with the miRNA inhibitor.

Apoptosis and necrosis assays. Mesothelioma cells were incubated with the miRNA or control inhibitor in 96-well plates for $24 \mathrm{~h}$, and the RealTime Glo Annexin V Apoptosis Assay reagent (Promega) was added to the cells after transfection. Relative levels of apoptosis and necrosis were measured by analyzing luminescence and fluorescence with a GloMax microplate reader according to the manufacturer's recommended protocol.

Chemosensitivity to pemetrexed and cisplatin. Mesothelioma cells transfected with the miRNA or control inhibitor were seeded into 96-well plates containing 0 to $50 \mu \mathrm{M}$ cisplatin or 0-100 $\mu \mathrm{M}$ pemetrexed (both purchased from Fujifilm Wako Pure Chemical Corporation). Viable cells were measured with the Cell Titer Glo 2.0 reagent (Promega) $72 \mathrm{~h}$ after the addition of chemical agents.

Reverse transcription-quantitative polymerase chain reaction (RT-qPCR). RNA was extracted from cells transfected with the miRNA inhibitor or control using a Maxwell RSC simplyRNA Cells kit and analyzed on a Maxwell RSC Instrument (Promega) according to the manufacturer's instructions. The extracted RNA was reverse-transcribed with SuperScript IV VILO Master Mix (Thermo Fisher Scientific) and amplified using PowerUp SYBR Green Master Mix (Thermo Fisher Scientific) on an AriaMax Real-Time PCR System (Agilent Technologies). Relative expression levels were calculated according to the comparative CT $(\Delta \Delta \mathrm{CT})$ method (17). Expression levels were normalized against the expression level of glyceraldehyde 3-phosphate dehydrogenase (GAPDH). The following primers were used: CDKN2D forward, 5'-TCACACTGCTGTGGTCAGCTTT-3', reverse, 5'-AGGATGTCCACGAGGTCCTGA-3', GAPDH forward, 5'-ACAACTTTTGGTATCATGGAAGG-3', and reverse, 5'-GCCATCACGCCACAGTTTC-3'.

Statistical analysis. All experiments were conducted at least three times. Experimental data are presented as means \pm standard deviation. Statistical significance of differences between two groups was analyzed with an unpaired Student's t-test. Statistical significance was set at $\mathrm{P}<0.05$.

\section{Results}

Inhibition of miR-18a reduces mesothelioma cell proliferation. Inhibition of miR-18a significantly decreased proliferation of mesothelioma cells compared to that by the negative control inhibitor in all four cell lines (Fig. 1A). After 3 days, inhibition of miR-18a significantly reduced viability by $42.3 \%$ in ACC-MESO1, 33.5\% in ACC-MESO4, 32.9\% in CRL-5915, and $27.5 \%$ in CRL-5946 cells. The role of miR-18a in mesothelioma cell proliferation was also evaluated by the colony formation assay (Fig. 1B and C). Inhibition of miR-18a significantly reduced the colony forming ability of all four cell lines.

miR-18a inhibition upregulates CDKN2D expression in mesothelioma cell lines. To understand the mechanism of miR-18a in inhibiting mesothelioma cell growth, we searched for its target gene using the online miRNA target database, Target Scan Human 7.2 (www.targetscan.org). CDKN2D was identified as a target gene of miR-18a. Furthermore, RT-qPCR analysis showed that inhibition of miR-18a significantly upregulated the expression of $C D K N 2 D$ (Fig. 2).

Inhibition of miR-18a reduces mesothelioma cell migration. Mesothelioma cells transfected with the miR-18a inhibitor exhibited lower migration rates compared to those transfected with the negative control inhibitor in all four cell lines (Fig. 3). At $24 \mathrm{~h}$, inhibition of miR-18a reduced the migration of ACC-MESO1 cells by $41.0 \%$, and at $48 \mathrm{~h}$ inhibition of miR-18a reduced the migration of ACC-MESO4, CRL-5915, 
A
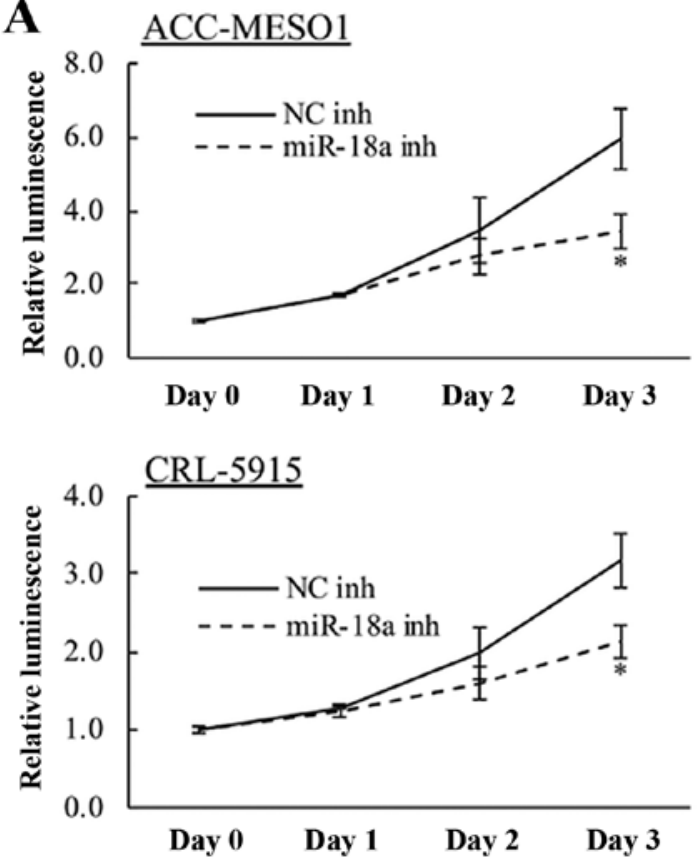

B

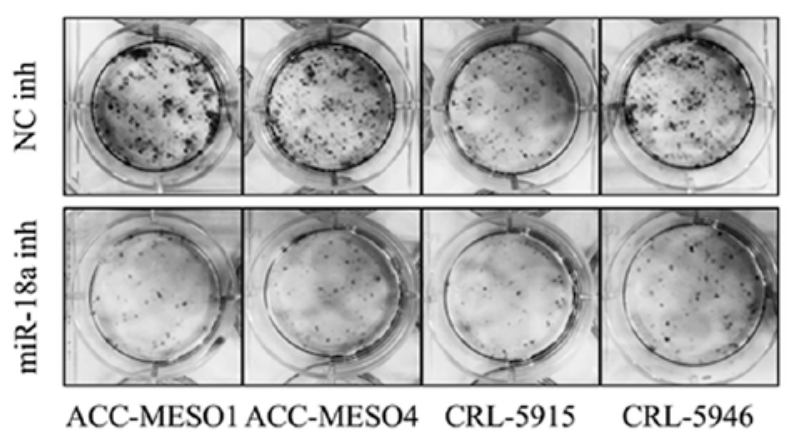

$\underline{\mathrm{ACC}-\mathrm{MESO} 4}$

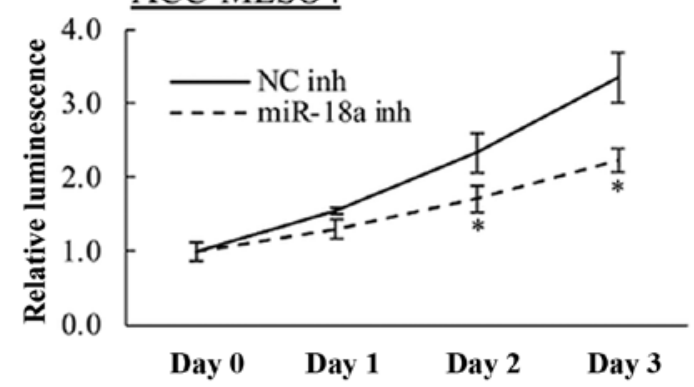

$\underline{\text { CRL-5946 }}$

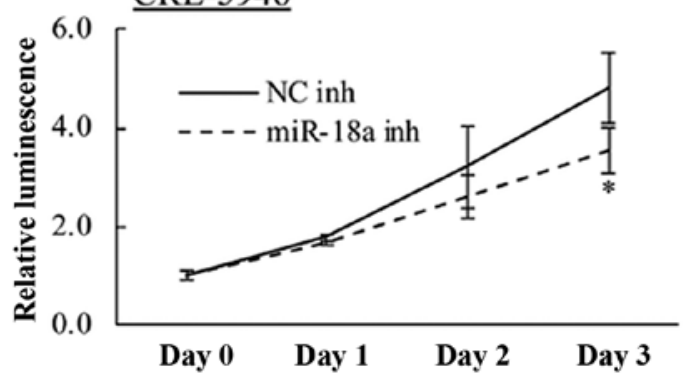

C

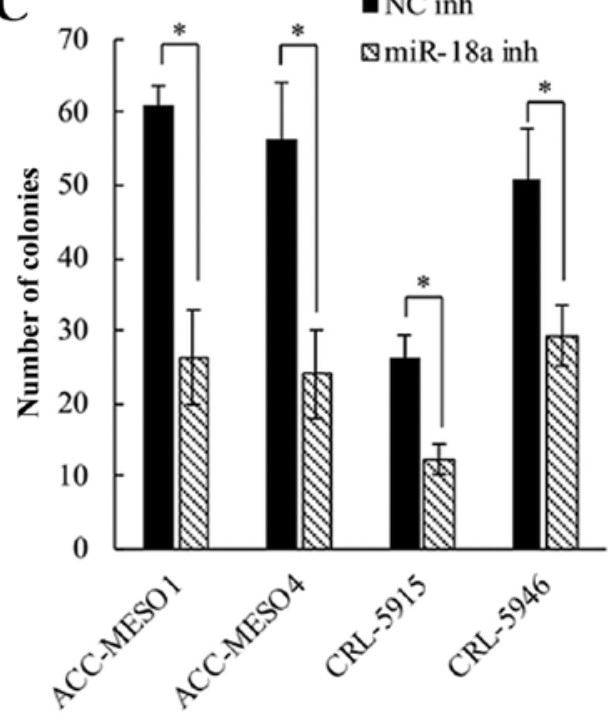

Figure 1. Cell proliferation and colony formation assay. (A) Mesothelioma cells (ACC-MESO1, ACC-MESO4, CRL-5915 and CRL-5946) transfected with the miR-18a inh exhibited lower proliferation rates compared with cells transfected with a NC inh. ${ }^{*} \mathrm{P}<0.05$ vs. NC inh. (B) Representative images of the colony formation assay. (C) Inhibition of miR-18a reduces the colony forming ability in four mesothelioma cell lines. "P<0.05. miR-18a inh, microRNA-18a inhibitor; $\mathrm{NC}$ inh, negative control inhibitor.

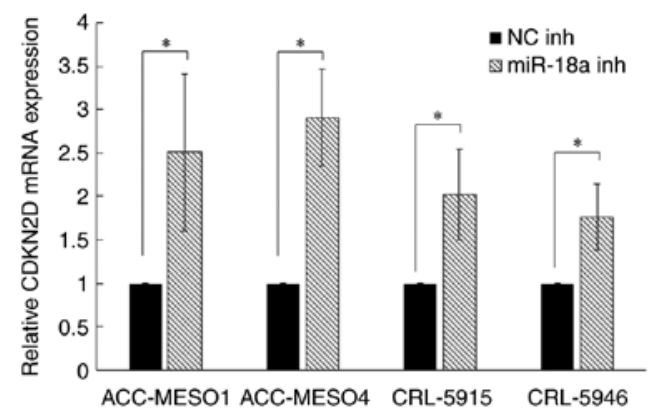

Figure 2. Reverse transcription-quantitative polymerase chain reaction analysis. CDKN2D mRNA was upregulated in mesothelioma cells transfected with miR-18a inh compared with in cells transfected with $\mathrm{NC}$ inh. ${ }^{*} \mathrm{P}<0.05$. miR-18a inh, microRNA-18a inhibitor; NC inh, negative control inhibitor. and CRL-5946 cells by 50.5, 53.0, and $33.7 \%$, respectively. Mesothelioma cell invasion was not significantly changed by inhibiting miR-18a (data not shown).

Inhibition of miR-18a increases apoptosis, but not necrosis, in mesothelioma cell lines. Transfection of the miR-18a inhibitor significantly increased the extent of apoptosis compared to that caused by the negative control inhibitor (Fig. 4A). Notably, ACC-MESO4 cells transfected with the miR-18a inhibitor exhibited over a three times increase in apoptosis compared to cells transfected with the negative control. However, no obvious change was observed in the extent of necrosis (Fig. 4B). 

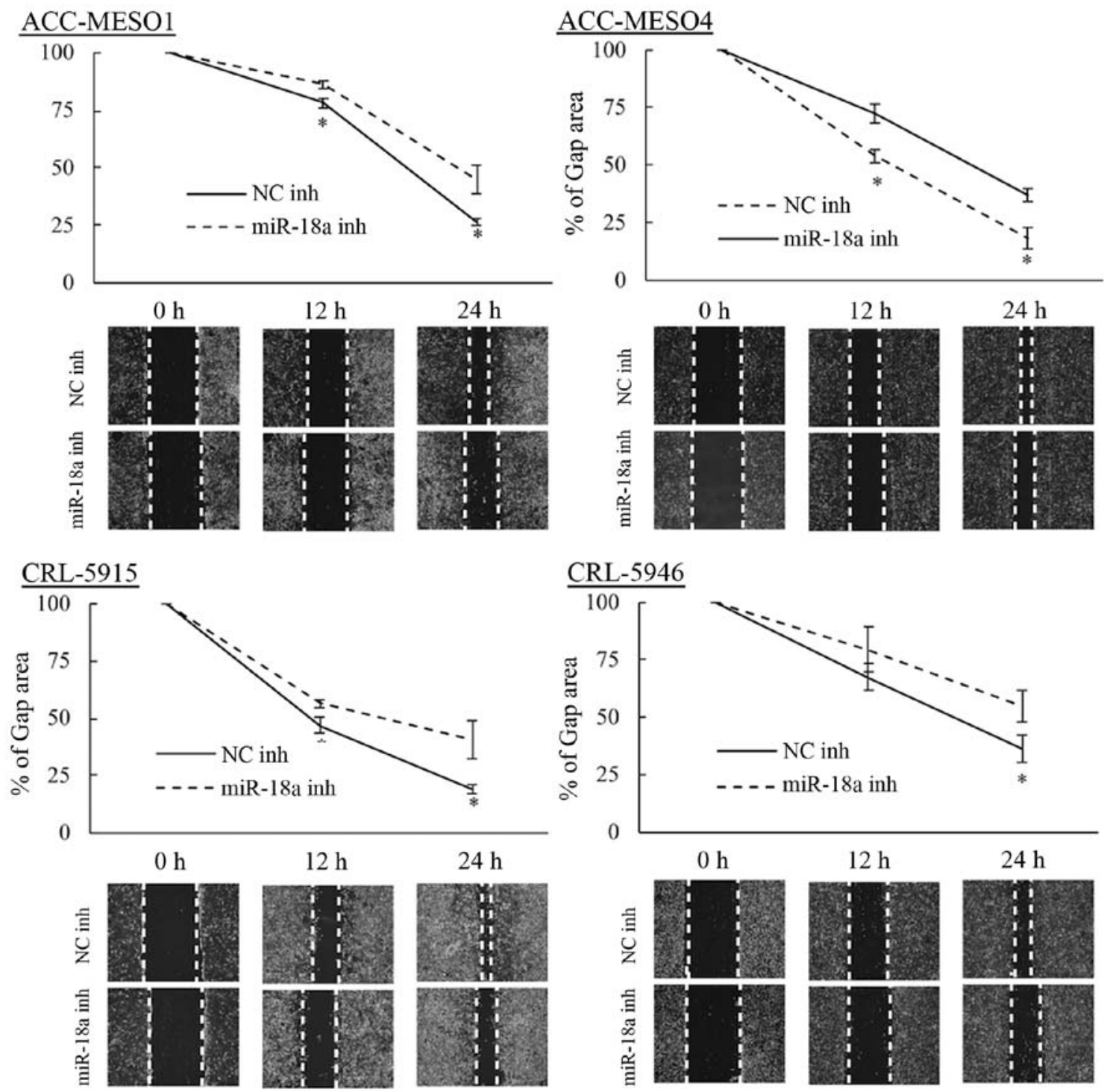

Figure 3. Wound scratch assay. Microscopy images representative of the wound gaps at 0, 12 and $24 \mathrm{~h}$ (ACC-MESO1 cells), or 0, 24 and $48 \mathrm{~h}$ (ACC-MESO4, CRL-5915 and CRL-5946 cells). Line graphs show reduced migration rates in cells transfected with miR-18a inh compared with in cells transfected with $\mathrm{NC}$ inh for all mesothelioma cell lines. All images were captured using an inverted microscope with a $4 \mathrm{X}$ objective. "P<0.05 vs. NC inh. miR-18a inh, microRNA-18a inhibitor; NC inh, negative control inhibitor.

Inhibition of miR-18a increases the sensitivity of mesothelioma cells to cisplatin, but not pemetrexed. In the chemosensitivity assay, CRL-5915 cells were more sensitive to both cisplatin and pemetrexed than the other three cell lines (ACC-MESO1, ACC-MESO4, and CRL-5946). We also found that transfection with the miR-18a inhibitor significantly enhanced sensitivity to cisplatin independent of the original sensitivity (Fig. 5A). At $0.5 \mu \mathrm{M}$ cisplatin, transfection of the miR-18a inhibitor reduced viability by $10.9,16.0,20.6$, and 16.3\% in ACC-MESO1, ACC-MESO4, CRL-5915, and CRL-5946 cells, respectively (statistically significant in ACC-MESO4, CRL-5915, and CRL-5946 cells). At $5 \mu \mathrm{M}$ cisplatin, transfection of the miR-18a inhibitor reduced viability by $20.5,23.3,20.5$, and $19.7 \%$ in ACC-MESO1, ACC-MESO4, CRL-5915, and CRL-5946 cells, respectively (statistically significant in all four cell lines). In this experiment, no obvious change in pemetrexed sensitivity was observed by transfection of the miR-18a inhibitor in cell lines relatively sensitive or resistant to this drug (Fig. 5B).

\section{Discussion}

miRNAs are short, non-coding RNAs that perform a variety of functions through incomplete binding to the 3'-untranslated region of a target gene (18). Because many miRNAs regulate important cell functions, such as proliferation and invasion, some have been researched as therapeutic agents for various human malignancies (19).

Several studies have focused on the role of miRNAs in the progression of mesothelioma cells $(20,21)$. Johnson et al (22) found that a miR-137 mimic inhibited the proliferation, invasion, and migration of mesothelioma cells, and Williams et al (23) found that miR-13 reduced proliferation, and increased apoptosis and necrosis of mesothelioma cells by downregulating MCL1. Further, we demonstrated previously 
A
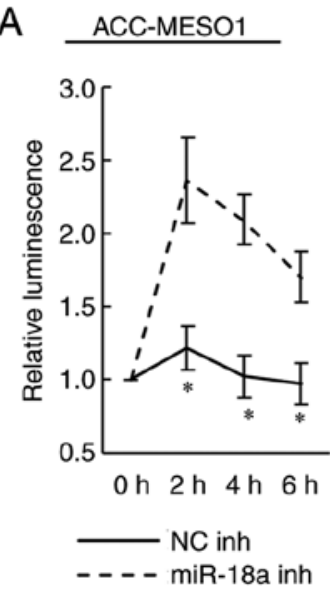

B

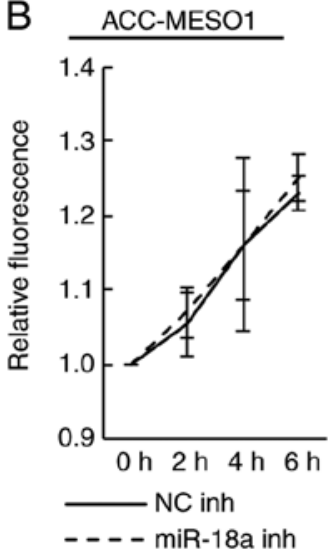

ACC-MESO4
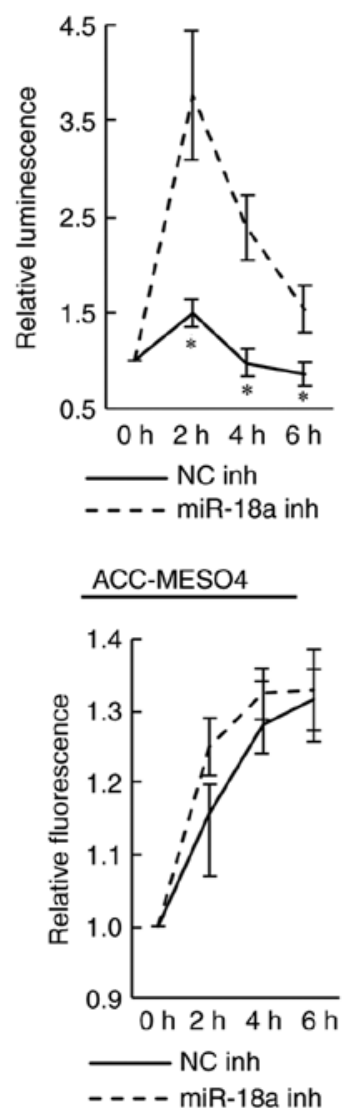
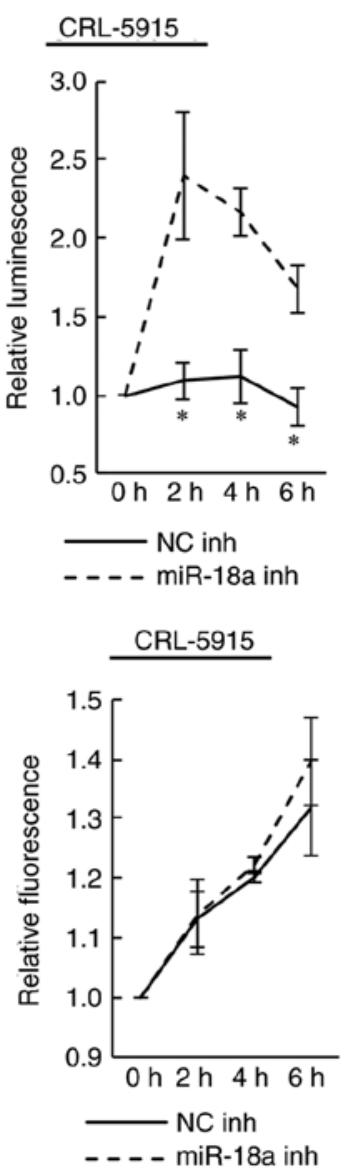

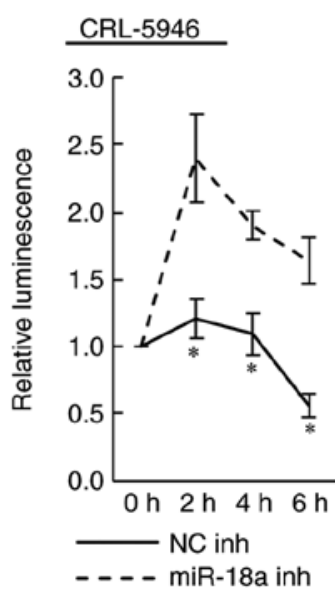

CRL-5946

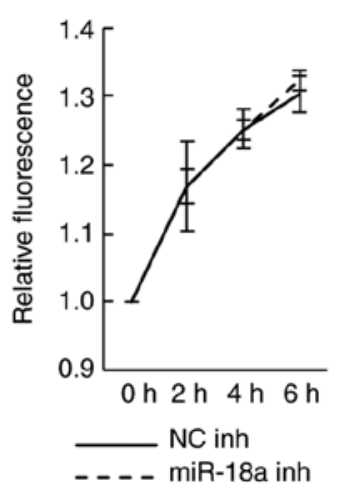

Figure 4. Apoptosis and necrosis assays. (A) Extent of apoptosis of mesothelioma cells transfected with miR-18a inh was significantly increased compared with cells transfected with NC inh. ${ }^{*} \mathrm{P}<0.05$ vs. NC inh. (B) No significant change in necrosis was observed between mesothelioma cells transfected with miR-18a inh and NC inh. miR-18a inh, microRNA-18a inhibitor; NC inh, negative control inhibitor.

that miR-1 and miR-214 inhibited mesothelioma cell progression by suppressing PIM1 (11,24), and miR-182 and miR-183 promoted mesothelioma cell progression by suppressing FOXO1 (25).

In our previous comprehensive analysis of miRNA expression by RT-qPCR using TaqMan Low Density Array Human miRNA Cards, both miR-18a-3p and miR-18a-5p were upregulated in malignant mesothelioma cell lines (ACC-MESO1, ACC-MESO4, CRL-2081, CRL-5820, CRL-5826, CRL-5915, and CRL-5946) compared to non-neoplastic mesothelial tissues (11). He et al (26) found that miR-18a-5p promoted mesothelioma cell proliferation by downregulating PIAS3, but little is known about the function of miR-18a-3p in mesothelioma cells. In the present study, we showed that inhibition of miR-18a-3p reduced proliferation and migration, but increased apoptosis of mesothelioma cells. miR-16 is the most extensively investigated miRNA in malignant mesothelioma and inhibits mesothelioma cell growth and enhances sensitivity to epidermal growth factor receptor inhibition (27). A clinical phase 1 trial using TargomiR, a miR-16-based miRNA mimic that targets the epidermal growth factor receptor, has been conducted. The objective partial response rate observed was $5 \%$ and the stable disease rate $67 \%$ (28). Thus, miRNA-based therapeutic targeting of malignant mesothelioma is promising.
In this study, we found that $C D K N 2 D$, a target gene of miR-18a-3p in malignant mesothelioma, was upregulated. The $C D K N 2 D$ gene $\left(\mathrm{p} 19^{\mathrm{INK} 4 \mathrm{~d}}\right)$ is located on chromosome $19 \mathrm{p} 13$, and its gene product negatively regulates the cell cycle by preventing the activation of CDK4 and CDK6 (29,30). Thus, CDK4 represses the proliferation of non-small cell lung cancer (31). However, further research on the target genes is needed to understand the mechanism of miR-18a-3p in terms of migration, apoptosis, and chemosensitivity to cisplatin in malignant mesothelioma.

Recent studies have demonstrated that some miRNAs play important roles, not only in cellular proliferation and invasion, but also in chemosensitivity. For example, miR-362-5p and miR-613 suppress chemosensitivity to cisplatin in gastric cancer $(32,33)$. Additionally, a correlation between the expression levels of miR-25, miR-145, and miR-210, and the effectiveness of pemetrexed maintenance treatment in lung adenocarcinoma, has been observed (34). Current standard chemotherapy for advanced stage malignant mesothelioma includes a combination of pemetrexed and cisplatin, but median survival remains only about 12 months, even though there is an approximately $40 \%$ response rate (6). This suggests that mesothelioma cells have chemoresistance to these anticancer agents. Moody et al (35) demonstrated that the loss of miR-31 enhanced sensitivity of malignant mesothelioma cells to cisplatin. Moreover, 
A
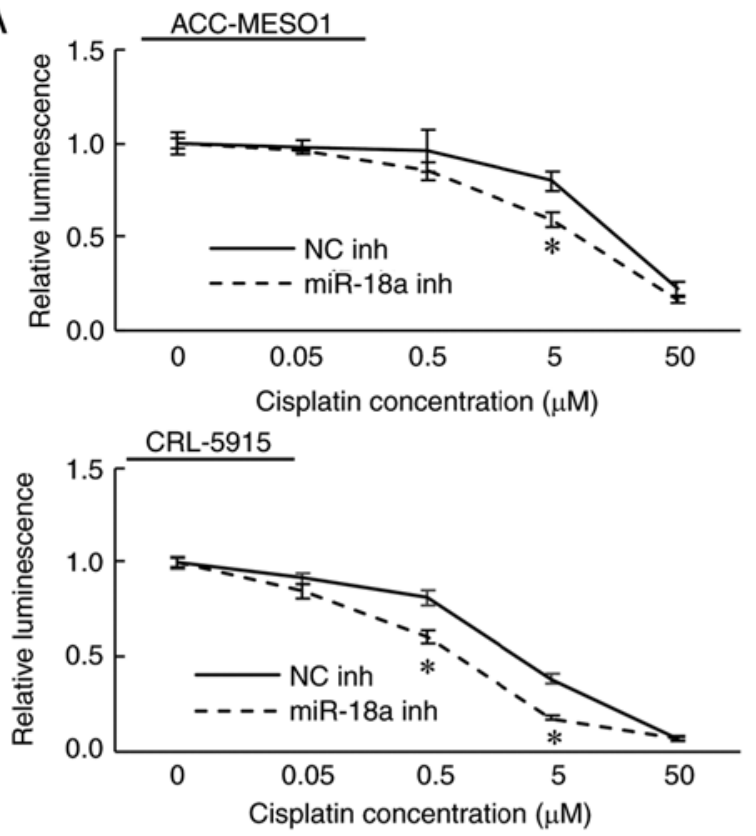

B
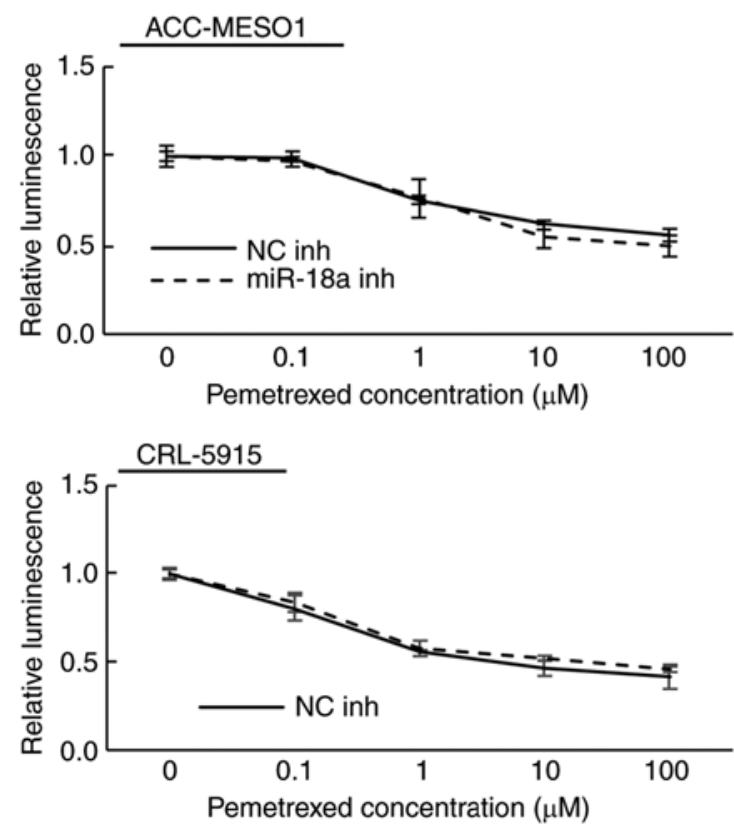
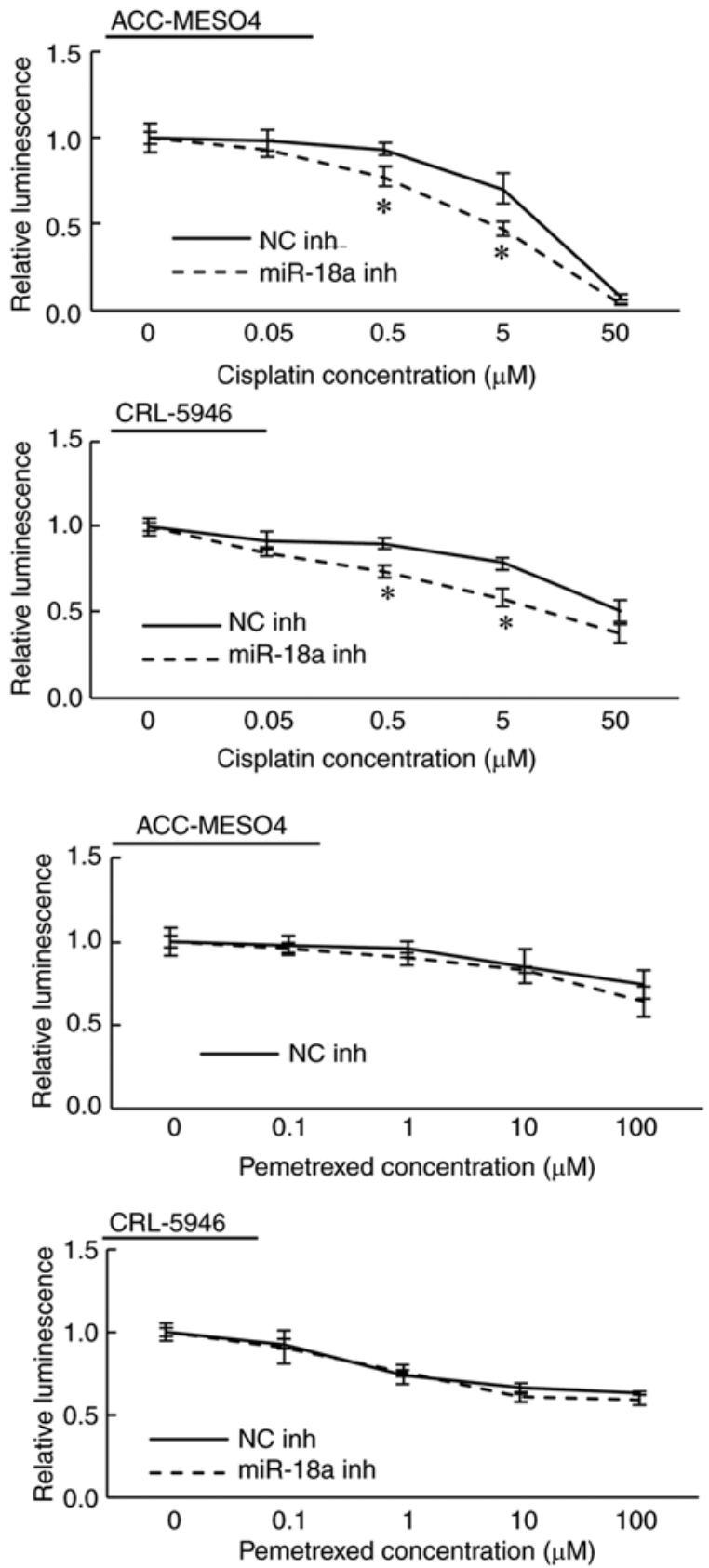

Figure 5. Chemosensitivity to cisplatin and pemetrexed. (A) Viability of mesothelioma cells transfected with miR-18a inh or NC inh $72 \mathrm{~h}$ after the addition of cisplatin (0-50 $\mu \mathrm{M})$. Transfection with miR-18a inh caused significant increases in sensitivity to 0.5 and $5 \mu \mathrm{M}$ cisplatin in ACC-MESO4, CRL-5915 and CRL-5946 cells, and $5 \mu \mathrm{M}$ cisplatin in ACC-MESO1 cells, compared with cells transfected with NC inh. * P<0.05 vs. NC inh. (B) Viability of mesothelioma cells transfected with miR-18a inh or NC inh measured $72 \mathrm{~h}$ after the addition of pemetrexed $(0-100 \mu \mathrm{M})$. Cell lines transfected with miR-18a inh exhibited no significant change in pemetrexed sensitivity compared with those transfected with NC inh. miR-18a inh, microRNA-18a inhibitor; NC inh, negative control inhibitor.

transfection of miR-145 and miR-379/411 induced chemosensitivity to pemetrexed in mesothelioma $(36,37)$. In the current study, we found that inhibition of miR-18a enhanced chemosensitivity to cisplatin in all four cell lines tested, including ACC-MESO1, ACC-MESO4, and CRL-5946 cells that showed only a slight decrease in viability at $5 \mu \mathrm{M}$. These results indicate that miR-18a targeted therapy may have benefits for patients with cisplatin-resistant mesothelioma. However, to clarify the detailed mechanism of miR-18a-3p in mesothelioma cell progression and chemosensitivity, further research is needed.
In conclusion, this study demonstrated that inhibition of miR-18a-3p significantly reduced mesothelioma progression and promoted chemosensitivity to cisplatin. Therefore, miR-18a is a potential therapeutic target for malignant mesothelioma.

\section{Acknowledgements}

The authors would like to thank Ms. Yukari Go and Mr. Tatsuya Nakagawa (Department of Pathology, Hiroshima University, Hiroshima, Japan) for their excellent 
technical assistance, and Ms Naomi Fukuhara (Department of Pathology, Hiroshima University, Hiroshima, Japan) for administrative support.

\section{Funding}

No funding was received.

\section{Availability of data and materials}

The datasets used and/or analyzed during the current study are available from the corresponding author on reasonable request.

\section{Authors' contributions}

RS, VJA and YT designed the study. VJA and YT supervised and facilitated the study. RS, KK, YK, TK and YF performed the experiments. RS analyzed the data. RS and VJA interpreted the results and wrote the manuscript. All authors read and approved the final manuscript.

\section{Ethics approval and consent to participate}

Not applicable.

\section{Patient consent for publication}

Not applicable.

\section{Competing interests}

The authors declare that they have no competing interests.

\section{References}

1. Frost G: The latency period of mesothelioma among a cohort of British asbestos workers (1978-2005). Br J Cancer 109 1965-1973, 2013.

2. Peto J, Decarli A, La Vecchia C, Levi F and Negri E: The European mesothelioma epidemic. Br J Cancer 79: 666-672, 1999.

3. Myojin T, Azuma K, Okumura J and Uchiyama I: Future trends of mesothelioma mortality in Japan based on a risk function. Ind Health 50: 197-204, 2012.

4. Keshava HB, Tang A, Siddiqui HU, Raja S, Raymond DP, Bribriesco A, Stevenson J, Murthy SC and Ahmad U: Largely Unchanged annual incidence and overall survival of pleural mesothelioma in the USA. World J Surg 43: 3239-3247, 2019.

5. Joshi TK and Gupta RK: Asbestos in developing countries: Magnitude of risk and its practical implications. Int J Occup Med Environ Health 17: 179-185, 2004.

6. Vogelzang NJ, Rusthoven JJ, Symanowski J, Denham C, Kaukel E, Ruffie P, Gatzemeier U, Boyer M, Emri S, Manegold C, et al: Phase III study of pemetrexed in combination with cisplatin versus cisplatin alone in patients with malignant pleural mesothelioma. J Clin Oncol 21: 2636-2644, 2003.

7. Bartel DP: MicroRNAs: Genomics, biogenesis, mechanism, and function. Cell 116: 281-297, 2004.

8. Wu KL, Tsai YM, Lien CT, Kuo PL and Hung AJ: The roles of MicroRNA in lung cancer. Int J Mol Sci 20: E1611, 2019.

9. Chen SN, Chang R, Lin LT, Chern CU, Tsai HW, Wen ZH, Li YH, Li CJ and Tsui KH: MicroRNA in ovarian cancer: Biology, pathogenesis, and therapeutic opportunities. Int J Environ Res Public Health 16: E1510, 2019.

10. Banelli B, Forlani A, Allemanni G, Morabito A, Pistillo MP and Romani M: MicroRNA in glioblastoma: An overview. Int J Genomics 2017: 7639084, 2017.
11. Amatya VJ, Mawas AS, Kushitani K, Mohi El-Din MM and Takeshima Y: Differential microRNA expression profiling of mesothelioma and expression analysis of miR-1 and miR-214 in mesothelioma. Int J Oncol 48: 1599-1607, 2016.

12. Song Y, Wang P, Zhao W, Yao Y, Liu X, Ma J, Xue Y and Liu Y: miR-18a regulates the proliferation, migration and invasion of human glioblastoma cell by targeting neogenin. Exp Cell Res 324: 54-64, 2014.

13. Zhang W, Lei C, Fan J and Wang J: miR-18a promotes cell proliferation of esophageal squamous cell carcinoma cells by increasing cylin D1 via regulating PTEN-PI3K-AKT-mTOR signaling axis. Biochem Biophys Res Commun 477: 144-149, 2016.

14. Xiao H, Liu Y, Liang P, Wang B, Tan H, Zhang Y, Gao X and Gao J: TP53TG1 enhances cisplatin sensitivity of non-small cell lung cancer cells through regulating miR-18a/PTEN axis. Cell Biosci 8: 23, 2018

15. Usami N, Fukui T, Kondo M, Taniguchi T, Yokoyama $\mathrm{T}$, Mori S, Yokoi K, Horio Y, Shimokata K, Sekido Y and Hida T: Establishment and characterization of four malignant pleural mesothelioma cell lines from Japanese patients. Cancer Sci 97: 387-394, 2006.

16. Gebäck T, Schulz MM, Koumoutsakos P and Detmar M: TScratch: A novel and simple software tool for automated analysis of monolayer wound healing assays. Biotechniques 46: 265-274, 2009

17. Livak KJ and Schmittgen TD: Analysis of relative gene expression data using real-time quantitative PCR and the 2(-Delta Delta C(T)) method. Methods 25: 402-408, 2001.

18. Ventura A and Jacks T: MicroRNAs and cancer: Short RNAs go a long way. Cell 136: 586-591, 2009.

19. Hanna J, Hossain GS and Kocerha J: The potential for microRNA therapeutics and clinical research. Front Genet 10: 478, 2019.

20. Truini A, Coco S, Genova C, Mora M, Dal Bello MG, Vanni I, Alama A, Rijavec E, Barletta G, Biello F, et al: Prognostic and therapeutic implications of MicroRNA in malignant pleural mesothelioma. Microrna 5: 12-18, 2016.

21. Lo Russo G, Tessari A, Capece M, Galli G, de Braud F, Garassino MC and Palmieri D: MicroRNAs for the diagnosis and management of malignant pleural mesothelioma: A literature review. Front Oncol 8: 650, 2018.

22. Johnson TG, Schelch K, Cheng YY, Williams M, Sarun KH, Kirschner MB, Kao S, Linton A, Klebe S, McCaughan BC, et al: Dysregulated expression of the microRNA miR-137 and its target YBX1 contribute to the invasive characteristics of malignant pleural mesothelioma. J Thorac Oncol 13: 258-272, 2018.

23. Williams M, Kirschner MB, Cheng YY, Hanh J, Weiss J, Mugridge N, Wright CM, Linton A, Kao SC, Edelman JJ, et al: miR-193a-3p is a potential tumor suppressor in malignant pleural mesothelioma. Oncotarget 6: 23480-23495, 2015.

24. Mawas AS, Amatya VJ, Suzuki R, Kushitani K, Mohi El-Din MM and Takeshima Y: PIM1 knockdown inhibits cell proliferation and invasion of mesothelioma cells. Int J Oncol 50: 1029-1034, 2017.

25. Suzuki R, Amatya VJ, Kushitani K, Kai Y, Kambara T and Takeshima Y: miR-182 and miR-183 promote cell proliferation and invasion by targeting FOXO1 in mesothelioma. Front Oncol 8: 446, 2018.

26. He T, McColl K, Sakre N, Chen Y, Wildey G and Dowlati A: Post-transcriptional regulation of PIAS3 expression by miR-18a in malignant mesothelioma. Mol Oncol 12: 2124-2135, 2018.

27. Schelch K, Kirschner MB, Williams M, Cheng YY, van Zandwijk N, Grusch M and Reid G: A link between the fibroblast growth factor axis and the miR-16 family reveals potential new treatment combinations in mesothelioma. Mol Oncol 12: 58-73, 2018.

28. van Zandwijk N, Pavlakis N, Kao SC, Linton A, Boyer MJ, Clarke S, Huynh Y, Chrzanowska A, Fulham MJ, Bailey DL, et al: Safety and activity of microRNA-loaded minicells in patients with recurrent malignant pleural mesothelioma: A first-in-man, phase 1, open-label, dose-escalation study. Lancet Oncol 18: 1386-1396, 2017.

29. Roussel MF: The INK4 family of cell cycle inhibitors in cancer. Oncogene 18: 5311-5317, 1999.

30. Bartkova J, Rajpert-De Meyts E, Skakkebaek NE, Lukas J and Bartek J: Deregulation of the G1/S-phase control in human testicular germ cell tumours. APMIS 111: 252-265; discussion 265-256, 2003.

31. Lin S, Wang MJ and Tseng KY: Polypyrimidine tract-binding protein induces p19(Ink4d) expression and inhibits the proliferation of H1299 cells. PLoS One 8: e58227, 2013. 
32. Wei X, Gao M, Ahmed Y, Gao M, Liu W, Zhang Y, Xie X, Zhao Q, Wang $\mathrm{H}$ and $\mathrm{Gu} \mathrm{K}$ : MicroRNA-362-5p enhances the cisplatin sensitivity of gastric cancer cells by targeting suppressor of zeste 12 protein. Oncol Lett 18: 1607-1616, 2019.

33. Xue M, Li G, Sun P, Zhang D, Fang X and Li W: MicroRNA-613 induces the sensitivity of gastric cancer cells to cisplatin through targeting SOX9 expression. Am J Transl Res 11: 885-894, 2019.

34. Shi SB, Wang M, Tian J, Li R, Chang CX and Qi JL: MicroRNA 25 , microRNA 145 , and microRNA 210 as biomarkers for predicting the efficacy of maintenance treatment with pemetrexed in lung adenocarcinoma patients who are negative for epidermal growth factor receptor mutations or anaplastic lymphoma kinase translocations. Transl Res 170: 1-7, 2016.
35. Moody HL, Lind MJ and Maher SG: MicroRNA-31 regulates chemosensitivity in malignant pleural mesothelioma. Mol Ther Nucleic Acids 8: 317-329, 2017.

36. Cioce M, Ganci F, Canu V, Sacconi A, Mori F, Canino C, Korita E, Casini B, Alessandrini G, Cambria A, et al: Protumorigenic effects of mir-145 loss in malignant pleural mesothelioma. Oncogene 33: 5319-5331, 2014.

37. Yamamoto K, Seike M, Takeuchi S, Soeno C, Miyanaga A, Noro R, Minegishi Y, Kubota K and Gemma A: miR-379/411 cluster regulates IL-18 and contributes to drug resistance in malignant pleural mesothelioma. Oncol Rep 32: 2365-2372, 2014. 